\title{
Fruit and seed traits of Berberis croatica Horvat and Berberis vulgaris $\mathbf{L}$.
}

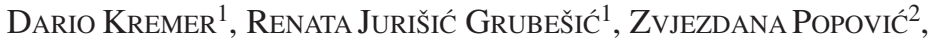 \\ KSENIJA KARLOVIĆ ${ }^{3 *}$ \\ ${ }^{1}$ Faculty of Pharmacy and Biochemistry, University of Zagreb, Schrottova 39, \\ HR-10000 Zagreb, Croatia. \\ ${ }^{2}$ Public institution »Nature Park Biokovo«, Trg Tina Ujevića 1, HR-21300 Makarska, \\ Croatia. \\ ${ }^{3}$ Faculty of Agriculture, University of Zagreb, Svetošimunska 25, HR-10000 Zagreb, \\ Croatia.
}

\begin{abstract}
The three year variability of fruits and seeds was investigated in Berberis croatica (Vošac, Mt Biokovo, Rakov Potok near Zagreb) and in B. vulgaris (Fran Kušan Pharmaceutical Botanical Garden in Zagreb). Berberis croatica had the following dimensions of fruits (seeds): length 7.28-7.88 (4.57-5.03) mm; width 3.85-3.99 (width 1: 2.06-2.20; width 2: 1.44-1.63) mm; weight 0.065-0.078 (0.0116-0.0134) g. Dimensions of B. vulgaris fruits (seeds) were: length 10.20-11.29 (5.71-6.24) mm; width 5.29-5.83 (width 1: 2.40-2.71; width 2: 1.60-1.98) mm; weight 0.1602-0.2199 (0.0146-0.0235) g. The fruit shape of both species was similar and the length/width ratio was 1.91-2.04 in $B$. croatica and 1.77-2.07 in B. vulgaris. The number of seeds per fruit was 1.23-1.58 in $B$. croatica and 1.36-1.54 in B. vulgaris. Generally, fruits and seeds of B. vulgaris were significantly longer, wider and heavier than fruits and seeds of $B$. croatica. ANOVA showed significant statistical differences between populations for all analyzed fruit and seed traits while the species significantly differed in all traits, except in the fruit shape and number of seeds in fruit.
\end{abstract}

Keywords: Berberis croatica, Berberis vulgaris, fruit, seed

\section{Introduction}

Inter- and intraspecific variability in fruit and/or seed traits has been in the focus of interest of many authors (JORDANo 1984, HENDRIX and Sun 1989, WiLlson et al. 1990, Herrera 1992, ObESO and HERRERA 1994, ERIKSSON 1999) but only few of them address the genus Berberis. At the same time, when dealing with the fruit and seed properties of

\footnotetext{
* Corresponding author, e-mail: karlovic@agr.hr

Copyright $^{\circledR} 2012$ by Acta Botanica Croatica, the Faculty of Science, University of Zagreb. All rights reserved.
} 
barberry species in relation with interannual variation (HERRERA 1998, ALLEN and WILSON 1992), papers are mainly concentrated on the seed and/or fruit production. Even though data regarding basic fruit and seed related traits for some of the European species of the genus Berberis, namely B. vulgaris (Rudolf 1974, AKBUluT et al. 2009) and B. hispanica (OBESO and HERRERA 1994) could be found, those concerning B. croatica are lacking. $B$. croatica is a species with a still unresolved taxonomic status and is still not recognized as a distinctive species by the leading authorities on European flora. Several attempts have been made to crystallize the morphological (KARLOVIĆ et al. 2009) and chemical difference (Zovko KončIĆ et al. 2010) between B. croatica and B. vulgaris. However, fruit and seed characters have not been included in the analysis to date yet they could help in the characterization of the distinctive morphological traits of these species.

\section{Material and methods}

Fruits of Berberis croatica Horvat and B. vulgaris L. were randomly collected during three years (from 2008 to 2010). B. croatica fruits were collected in one natural habitat (Vošac, Mt Biokovo, in text as abbreviation Vo; $43^{\circ} 18^{\prime}$ N; $17^{\circ} 02^{\prime}$ E; $1422 \mathrm{~m}$ a.s.1.), while $B$. vulgaris fruits were collected in one natural (Rakov Potok near Zagreb, in text as abbreviation RP; 45 47' N; 1542' E; 160 m a.s.l.) and one cultivated habitat (Fran Kušan Pharmaceutical Botanical Garden, Zagreb, in text as abbreviation PBG; 4550' N; 1559’ E; 195 m a.s.l.). Twenty plants were harvested in the locality Vošac, ten in the locality Rakov Potok and six in the cultivated habitat. According to our permit from the Ministry of Culture of the Republic of Croatia, we were able to analyse 100 fruits for the locality Vošac and so the same number of B. vulgaris fruits (100) was collected for each population. Length, width and weight of fruits, as well as seeds, were measured. Since the seeds of the researched Berberis species have irregular shapes, the width of the seed was measured on two axes (width 1 and width 2). The ratio between length and width of fruits and length and width (width 1, width 2 ) of seeds was obtained through mathematical calculation, as well as their mutual (fruit-seed) ratio, which included length, width and weight. A greater ratio indicates narrower fruits (or seeds), while a smaller ratio indicates more orbicular fruits (or seeds).

The differences in fruit and seed traits between species and populations were analyzed by the General Linear Models (GLM) procedure using repeated measures ANOVA and Tuckey's post-hoc test at the $p \leq 0.05$ level. The statistical analysis was performed using Statistica 7 software (StatSoft Inc., Tulsa, OK, USA).

\section{Results}

Table 1 presents descriptive statistics for the analyzed traits of fruits and seeds of Berberis croatica and B. vulgaris. The average length of B. croatica fruits ranged from 7.28 $\mathrm{mm}$ (Vo 2010) to $7.88 \mathrm{~mm}$ (Vo 2009) while the width ranged from $3.85 \mathrm{~mm}$ (Vo 2010) to $3.99 \mathrm{~mm}$ (Vo 2008). Weight of B. croatica fruits ranged from $0.065 \mathrm{~g}$ (Vo 2008) to $0.078 \mathrm{~g}$ (Vo 2010). Fruits of B. vulgaris were the largest and their length ranged from $10.20 \mathrm{~mm}$ (PBG 2009) to $11.29 \mathrm{~mm}$ (RP 2010), while the width ranged from $5.29 \mathrm{~mm}$ (RP 2008) to $5.83 \mathrm{~mm}$ (PBG 2009). Weight of B. vulgaris fruits ranged from $0.1602 \mathrm{~g}$ (RP 2009) to $0.2199 \mathrm{~g}$ (PBG 2009). The shapes of the two species were similar and the ratio between 
length and width of fruits was from 1.91 (Vo 2010) to 2.04 (Vo 2009) in B. croatica and from 1.77 (PBG 2009) to 2.07 (RP 2010) in B. vulgaris. The most variable trait was fruit weight, ranging from $25.61 \%$ (Vo 2010) to $28.55 \%$ (Vo 2008) and from $22.84 \%$ (RP 2010) to $36.54 \%$ (PBG 2008) in B. croatica and B. vulgaris, respectively. The least variable trait was fruit length, which ranged from $5.84 \%$ (Vo 2009) to $9.62 \%$ (Vo 2010) and from $6.37 \%$ (PBG 2009) to $9.83 \%$ (RP 2008) in B. croatica and B. vulgaris, respectively (Tab. 1).

The number of seeds per fruit ranged from 1.23 (Vo 2010) to 1.58 (Vo 2009) in $B$. croatica and from 1.36 (RP 2008, RP 2010) to 1.54 (PBG 2010) in B. vulgaris.

Tab. 1. Statistics for analyzed traits of fruits and seeds of Berberis croatica - Vošac (Vo) and $B$. vulgaris - Rakov Potok (RP) and Pharmaceutical Botanical Garden »Fran Kušan« (PBG). Min, Max and Std. dev. are in mm (length, width) and g (weight); CV (coefficient of variability) is in \%. Minimum and maximum are bolded.

\begin{tabular}{|c|c|c|c|c|c|c|c|c|c|c|}
\hline & \multirow[b]{2}{*}{ Statistics } & \multicolumn{3}{|c|}{ B. croatica } & \multicolumn{6}{|c|}{ B. vulgaris } \\
\hline & & $\begin{array}{c}\text { Vo } \\
2008\end{array}$ & $\begin{array}{c}\text { Vo } \\
2009\end{array}$ & $\begin{array}{c}\text { Vo } \\
2010\end{array}$ & $\begin{array}{c}\mathrm{RP} \\
2008\end{array}$ & $\begin{array}{c}\mathrm{RP} \\
2009\end{array}$ & $\begin{array}{c}\text { RP } \\
2010\end{array}$ & $\begin{array}{l}\text { PBG } \\
2008\end{array}$ & $\begin{array}{l}\text { PBG } \\
2009\end{array}$ & $\begin{array}{l}\text { PBG } \\
2010\end{array}$ \\
\hline \multicolumn{11}{|c|}{ Fruit traits } \\
\hline \multirow[t]{6}{*}{ Length } & $\mathrm{N}$ & 100 & 100 & 100 & 100 & 100 & 100 & 100 & 100 & 100 \\
\hline & Mean & 7.83 & 7.88 & 7.28 & 10.89 & 10.39 & 11.29 & 10.42 & 10.20 & 10.41 \\
\hline & Min & 7.00 & 6.89 & 5.26 & 5.34 & 8.64 & 5.84 & 8.94 & 9.01 & 7.33 \\
\hline & Max & 9.04 & 9.07 & 9.43 & 12.74 & 12.10 & 13.26 & 12.54 & 12.47 & 12.12 \\
\hline & Std. dev. & 0.50 & 0.46 & 0.70 & 1.07 & 0.79 & 0.91 & 0.74 & 0.65 & 0.67 \\
\hline & $\mathrm{CV}$ & 6.39 & 5.84 & 9.62 & 9.83 & 7.60 & 8.06 & 7.10 & 6.37 & 6.44 \\
\hline \multirow[t]{6}{*}{ Width } & $\mathrm{N}$ & 100 & 100 & 100 & 100 & 100 & 100 & 100 & 100 & 100 \\
\hline & Mean & 3.99 & 3.91 & 3.85 & 5.29 & 5.30 & 5.55 & 5.42 & 5.83 & 5.71 \\
\hline & Min & 3.03 & 2.86 & 2.23 & 3.91 & 4.22 & 4.16 & 3.76 & 4.59 & 3.50 \\
\hline & Max & 5.03 & 5.06 & 5.01 & 6.90 & 6.90 & 7.04 & 7.76 & 7.58 & 7.43 \\
\hline & Std. dev. & 0.42 & 0.44 & 0.51 & 0.69 & 0.62 & 0.54 & 0.94 & 0.68 & 0.73 \\
\hline & $\mathrm{CV}$ & 10.53 & 11.25 & 13.25 & 13.04 & 11.70 & 9.73 & 17.34 & 11.66 & 12.78 \\
\hline \multirow{6}{*}{$\begin{array}{l}\text { Length/ } \\
\text { Width }\end{array}$} & $\mathrm{N}$ & 100 & 100 & 100 & 100 & 100 & 100 & 100 & 100 & 100 \\
\hline & Mean & 1.98 & 2.04 & 1.91 & 2.05 & 1.98 & 2.07 & 1.97 & 1.77 & 1.85 \\
\hline & Min & 1.46 & 1.52 & 1.40 & 1.22 & 1.55 & 1.30 & 1.49 & 1.47 & 1.12 \\
\hline & Max & 2.88 & 2.88 & 3.05 & 2.77 & 2.78 & 2.40 & 2.60 & 2.07 & 2.66 \\
\hline & Std. dev. & 0.25 & 0.25 & 0.22 & 0.19 & 0.22 & 0.19 & 0.30 & 0.16 & 0.25 \\
\hline & $\mathrm{CV}$ & 12.63 & 12.25 & 11.52 & 9.27 & 11.11 & 9.18 & 15.23 & 9.04 & 13.51 \\
\hline \multirow[t]{6}{*}{ Weight } & $\mathrm{N}$ & 100 & 100 & 100 & 100 & 100 & 100 & 100 & 100 & 100 \\
\hline & Mean & 0.065 & 0.071 & 0.078 & 0.182 & 0.160 & 0.209 & 0.180 & 0.220 & 0.206 \\
\hline & Min & 0.034 & 0.032 & 0.036 & 0.066 & 0.082 & 0.107 & 0.062 & 0.102 & 0.051 \\
\hline & Max & 0.119 & 0.113 & 0.132 & 0.307 & 0.250 & 0.328 & 0.362 & 0.376 & 0.348 \\
\hline & Std. dev. & 0.018 & 0.020 & 0.020 & 0.050 & 0.037 & 0.048 & 0.066 & 0.0531 & 0.062 \\
\hline & $\mathrm{CV}$ & 27.06 & 28.55 & 25.61 & 27.48 & 22.85 & 22.84 & 36.54 & 24.15 & 30.05 \\
\hline
\end{tabular}


Kremer D., Jurišić GrubeŠić R., Popović Z., Karlović K.

Tab. 1. - continued

\begin{tabular}{|c|c|c|c|c|c|c|c|c|c|c|}
\hline & \multirow[b]{2}{*}{ Statistics } & \multicolumn{3}{|c|}{ B. croatica } & \multicolumn{6}{|c|}{ B. vulgaris } \\
\hline & & $\begin{array}{c}\text { Vo } \\
2008\end{array}$ & $\begin{array}{c}\text { Vo } \\
2009\end{array}$ & $\begin{array}{c}\text { Vo } \\
2010\end{array}$ & $\begin{array}{c}\mathrm{RP} \\
2008\end{array}$ & $\begin{array}{c}\text { RP } \\
2009\end{array}$ & $\begin{array}{c}\mathrm{RP} \\
2010\end{array}$ & $\begin{array}{l}\text { PBG } \\
2008\end{array}$ & $\begin{array}{l}\text { PBG } \\
2009\end{array}$ & $\begin{array}{l}\text { PBG } \\
2010\end{array}$ \\
\hline \multicolumn{11}{|c|}{ Seed traits } \\
\hline \multirow[t]{6}{*}{ Length } & $\mathrm{N}$ & 145 & 158 & 123 & 136 & 138 & 136 & 142 & 149 & 154 \\
\hline & Mean & 5.03 & 4.92 & 4.57 & 5.88 & 5.71 & 5.99 & 6.14 & 5.94 & 6.24 \\
\hline & Min & 4.13 & 3.97 & 3.58 & 4.30 & 4.23 & 4.29 & 4.81 & 5.14 & 4.51 \\
\hline & Max & 5.72 & 5.84 & 5.74 & 6.94 & 6.88 & 6.99 & 7.29 & 6.64 & 7.75 \\
\hline & Std. dev. & 0.31 & 0.30 & 0.38 & 0.47 & 0.52 & 0.47 & 0.45 & 0.36 & 0.49 \\
\hline & $\mathrm{CV}$ & 6.16 & 6.10 & 8.32 & 7.99 & 9.11 & 7.85 & 7.33 & 6.06 & 7.85 \\
\hline \multirow[t]{6}{*}{ Width 1} & $\mathrm{~N}$ & 145 & 158 & 123 & 136 & 138 & 136 & 142 & 149 & 154 \\
\hline & Mean & 2.10 & 2.06 & 2.20 & 2.57 & 2.55 & 2.71 & 2.46 & 2.40 & 2.50 \\
\hline & Min & 1.28 & 1.22 & 1.30 & 1.43 & 1.54 & 2.03 & 1.78 & 1.87 & 1.68 \\
\hline & Max & 2.90 & 2.51 & 2.60 & 3.09 & 3.16 & 3.14 & 3.28 & 3.14 & 3.32 \\
\hline & Std. dev. & 0.26 & 0.23 & 0.18 & 0.27 & 0.35 & 0.23 & 0.33 & 0.32 & 0.31 \\
\hline & $\mathrm{CV}$ & 12.38 & 11.17 & 8.18 & 10.51 & 13.73 & 8.49 & 13.41 & 13.33 & 12.40 \\
\hline \multirow[t]{6}{*}{ Width 2} & $\mathrm{~N}$ & 145 & 158 & 123 & 136 & 138 & 136 & 142 & 149 & 154 \\
\hline & Mean & 1.49 & 1.44 & 1.63 & 1.94 & 1.82 & 1.98 & 1.61 & 1.60 & 1.66 \\
\hline & Min & 0.82 & 0.92 & 0.85 & 0.87 & 1.09 & 0.96 & 0.50 & 0.79 & 0.83 \\
\hline & Max & 2.27 & 1.97 & 1.96 & 2.48 & 2.75 & 2.57 & 2.36 & 2.24 & 2.66 \\
\hline & Std. dev. & 0.26 & 0.21 & 0.22 & 0.30 & 0.25 & 0.30 & 0.34 & 0.34 & 0.36 \\
\hline & $\mathrm{CV}$ & 17.45 & 14.58 & 13.50 & 15.46 & 13.74 & 15.15 & 21.12 & 21.25 & 21.69 \\
\hline Length/ & $\mathrm{N}$ & 145 & 158 & 123 & 136 & 138 & 136 & 142 & 149 & 154 \\
\hline \multirow[t]{5}{*}{ Width 1} & Mean & 2.43 & 2.41 & 2.09 & 2.30 & 2.26 & 2.22 & 2.53 & 2.50 & 2.52 \\
\hline & Min & 1.87 & 1.93 & 1.66 & 1.88 & 1.87 & 1.71 & 1.88 & 2.01 & 1.90 \\
\hline & Max & 3.62 & 3.53 & 2.83 & 3.30 & 2.96 & 2.67 & 3.40 & 3.07 & 3.96 \\
\hline & Std. dev. & 0.30 & 0.26 & 0.20 & 0.23 & 0.22 & 0.21 & 0.31 & 0.24 & 0.30 \\
\hline & $\mathrm{CV}$ & 12.35 & 10.79 & 9.57 & 10.00 & 9.73 & 9.46 & 12.25 & 9.60 & 11.90 \\
\hline Length/ & $\mathrm{N}$ & 145 & 158 & 123 & 136 & 138 & 136 & 142 & 149 & 154 \\
\hline \multirow[t]{5}{*}{ Width 2} & Mean & 3.48 & 3.48 & 2.86 & 3.12 & 3.18 & 3.10 & 3.95 & 3.88 & 3.89 \\
\hline & Min & 2.04 & 2.45 & 2.17 & 2.22 & 2.15 & 2.15 & 2.62 & 2.58 & 2.44 \\
\hline & Max & 5.09 & 4.95 & 5.01 & 5.65 & 4.71 & 5.46 & 6.11 & 6.67 & 6.72 \\
\hline & Std. dev. & 0.57 & 0.51 & 0.45 & 0.59 & 0.42 & 0.59 & 0.80 & 0.84 & 0.83 \\
\hline & $\mathrm{CV}$ & 16.38 & 14.66 & 15.73 & 18.91 & 13.21 & 19.03 & 20.25 & 21.65 & 21.34 \\
\hline Width 1/ & $\mathrm{N}$ & 145 & 158 & 123 & 136 & 138 & 136 & 142 & 149 & 154 \\
\hline \multirow[t]{5}{*}{ Width 2} & Mean & 1.45 & 1.46 & 1.37 & 1.35 & 1.42 & 1.40 & 1.58 & 1.56 & 1.55 \\
\hline & Min & 0.56 & 0.92 & 1.01 & 1.07 & 0.76 & 0.98 & 1.02 & 1.03 & 0.93 \\
\hline & Max & 2.12 & 1.91 & 1.89 & 2.40 & 2.20 & 2.29 & 2.51 & 2.96 & 2.81 \\
\hline & Std. dev. & 0.29 & 0.22 & 0.16 & 0.19 & 0.20 & 0.21 & 0.32 & 0.34 & 0.30 \\
\hline & $\mathrm{CV}$ & 20.00 & 15.07 & 11.68 & 14.07 & 14.08 & 15.00 & 20.25 & 21.79 & 19.35 \\
\hline
\end{tabular}


Tab. 1. - continued

\begin{tabular}{|c|c|c|c|c|c|c|c|c|c|c|}
\hline & \multirow[b]{2}{*}{ Statistics } & \multicolumn{3}{|c|}{ B. croatica } & \multicolumn{6}{|c|}{ B. vulgaris } \\
\hline & & $\begin{array}{c}\text { Vo } \\
2008\end{array}$ & $\begin{array}{c}\text { Vo } \\
2009\end{array}$ & $\begin{array}{c}\text { Vo } \\
2010\end{array}$ & $\begin{array}{c}\text { RP } \\
2008\end{array}$ & $\begin{array}{c}\text { RP } \\
2009\end{array}$ & $\begin{array}{c}\text { RP } \\
2010\end{array}$ & $\begin{array}{l}\text { PBG } \\
2008\end{array}$ & $\begin{array}{l}\text { PBG } \\
2009\end{array}$ & $\begin{array}{l}\text { PBG } \\
2010\end{array}$ \\
\hline \multirow[t]{6}{*}{ Weight } & $\mathrm{N}$ & 145 & 158 & 123 & 136 & 138 & 136 & 142 & 149 & 154 \\
\hline & Mean & 0.012 & 0.012 & 0.013 & 0.022 & 0.022 & 0.024 & 0.018 & 0.017 & 0.015 \\
\hline & Min & 0.001 & 0.001 & 0.005 & 0.004 & 0.007 & 0.010 & 0.003 & 0.003 & 0.002 \\
\hline & Max & 0.018 & 0.021 & 0.019 & 0.035 & 0.034 & 0.035 & 0.038 & 0.046 & 0.033 \\
\hline & Std. dev. & 0.003 & 0.003 & 0.003 & 0.007 & 0.013 & 0.005 & 0.008 & 0.007 & 0.006 \\
\hline & $\mathrm{CV}$ & 24.14 & 23.97 & 20.15 & 31.78 & 57.72 & 22.13 & 44.32 & 43.79 & 42.47 \\
\hline \multicolumn{11}{|c|}{ Seed and fruit traits } \\
\hline \multirow{6}{*}{$\begin{array}{l}\text { Seeds in } \\
\text { Fruit }\end{array}$} & $\mathrm{N}$ & 100 & 100 & 100 & 100 & 100 & 100 & 100 & 100 & 100 \\
\hline & Mean & 1.45 & 1.58 & 1.23 & 1.36 & 1.38 & 1.36 & 1.42 & 1.49 & 1.54 \\
\hline & Min & 1.00 & 1.00 & 1.00 & 1.00 & 1.00 & 1.00 & 1.00 & 1.00 & 1.00 \\
\hline & Max & 2.00 & 3.00 & 2.00 & 2.00 & 2.00 & 2.00 & 2.00 & 2.00 & 2.00 \\
\hline & Std. dev. & 0.50 & 0.52 & 0.42 & 0.48 & 0.49 & 0.48 & 0.50 & 0.50 & 0.50 \\
\hline & $\mathrm{CV}$ & 34.49 & 32.91 & 34.15 & 35.29 & 35.51 & 35.29 & 35.21 & 33.56 & 32.47 \\
\hline \multicolumn{2}{|c|}{ Seed/Fruit Length } & 0.64 & 0.62 & 0.63 & 0.54 & 0.55 & 0.53 & 0.59 & 0.60 & 0.60 \\
\hline \multicolumn{2}{|c|}{ Seed width $1 /$ Fruit Width } & 0.61 & 0.62 & 0.54 & 0.43 & 0.43 & 0.40 & 0.47 & 0.43 & 0.44 \\
\hline \multicolumn{2}{|c|}{ Seed width $2 /$ Fruit Width } & 0.87 & 0.89 & 0.74 & 0.59 & 0.60 & 0.56 & 0.73 & 0.67 & 0.68 \\
\hline \multicolumn{2}{|c|}{ Seed/Fruit Weight } & 0.177 & 0.170 & 0.172 & 0.119 & 0.137 & 0.113 & 0.098 & 0.077 & 0.071 \\
\hline
\end{tabular}

Seeds of $B$. vulgaris were also larger than seeds of $B$. croatica. The length of $B$. croatica seeds ranged from $4.57 \mathrm{~mm}$ (Vo 2010) to $5.03 \mathrm{~mm}$ (Vo 2008), width 1 from $2.06 \mathrm{~mm}$ (Vo 2009) to $2.20 \mathrm{~mm}$ (Vo 2010), width 2 from $1.44 \mathrm{~mm}$ (Vo 2009) to $1.63 \mathrm{~mm}$ (Vo 2010) and the weight from $0.0116 \mathrm{~g}$ (Vo 2008) to 0.0134 (Vo 2010) g. In B. vulgaris seeds the length ranged from $5.71 \mathrm{~mm}$ (RP 2009) to $6.24 \mathrm{~mm}$ (PBG 2010), width 1 from $2.40 \mathrm{~mm}$ (PBG 2009) to $2.71 \mathrm{~mm}$ (RP 2010), width 2 from $1.60 \mathrm{~mm}$ (PBG 20) to $1.98 \mathrm{~mm}$ (RP 2010) and the weight from $0.0146 \mathrm{~g}$ (PBG 2010) to $0.0235 \mathrm{~g}$ (RP 2010). The most variable trait was seed weight, ranging from $20.15 \%$ (Vo 2010) to $24.14 \%$ (Vo 2008) and from $22.13 \%$ (RP 2010) to $57.72 \%$ (RP 2009) in B. croatica and B. vulgaris, respectively. The least variable trait was seed length and it ranged from $6.10 \%$ (Vo 2009) to $8.32 \%$ (Vo 2010) and from $6.06 \%$ (PBG 2009) to $9.11 \%$ (RP 2009) in B. croatica and B. vulgaris, respectively (Tab. 1).

ANOVA showed significant statistical differences between species for most analyzed fruit and seed traits (Tab. 2). The only exception was the ratio between fruit length and width as well as the number of seeds in the fruit. The analysis of fruit traits between species showed that fruits of B. vulgaris were significantly longer, wider and heavier than fruits of B. croatica (Tab. 3). Nevertheless, fruit shape (based on ratio between length and wide) was similar in the two species. 
Kremer D., Jurišić GrubeŠić R., Popović Z., Karlović K.

Tab. 2. Results of ANOVA for analysed traits of fruit and seed. Asterisks indicate significant $p$ values at $p \leq 0.05$.

\begin{tabular}{|c|c|c|c|c|c|}
\hline Effect & $\begin{array}{l}\text { Sum of } \\
\text { Squares }\end{array}$ & $\begin{array}{l}\text { Degr. of } \\
\text { freedom }\end{array}$ & $\begin{array}{l}\text { Mean } \\
\text { Square }\end{array}$ & $\mathrm{F}$ & p-level \\
\hline \multicolumn{6}{|c|}{ Fruit Length } \\
\hline Population & 1765.34 & 2 & 882.67 & 1608.8 & $<0.0001 *$ \\
\hline Species & 1725.57 & 1 & 1725.57 & 2536.49 & $<0.0001 *$ \\
\hline \multicolumn{6}{|c|}{ Fruit Width } \\
\hline Population & 523.84 & 2 & 261.92 & 643.30 & $<0.0001 *$ \\
\hline Species & 513.08 & 1 & 513.08 & 1161.04 & $<0.0001 *$ \\
\hline \multicolumn{6}{|c|}{ Fruit Length/Width } \\
\hline Population & 4.504 & 2 & 2.252 & 40.52 & $<0.0001 *$ \\
\hline Species & 0.202 & 1 & 0.202 & 2.89 & 0.0904 \\
\hline \multicolumn{6}{|c|}{ Fruit Weight } \\
\hline Population & 2.99660 & 2 & 1.49830 & 832.78 & $<0.0001 *$ \\
\hline Species & 2.94489 & 1 & 2.94489 & 1497.422 & $<0.0001 *$ \\
\hline \multicolumn{6}{|c|}{ Seed Length } \\
\hline Population & 347.93 & 2 & 173.97 & 977.2 & $<0.0001 *$ \\
\hline Species & 335.54 & 1 & 335.54 & 1608.2 & $<0.0001 *$ \\
\hline \multicolumn{6}{|c|}{ Seed Width 1} \\
\hline Population & 49.319 & 2 & 24.659 & 300.74 & $<0.0001^{*}$ \\
\hline Species & 43.625 & 1 & 43.625 & 454.14 & $<0.0001 *$ \\
\hline \multicolumn{6}{|c|}{ Seed Width 2} \\
\hline Population & 31.795 & 2 & 15.898 & 189.02 & $<0.0001 *$ \\
\hline Species & 15.041 & 1 & 15.041 & 119.48 & $<0.0001 *$ \\
\hline \multicolumn{6}{|c|}{ Seed Length/Width 1} \\
\hline Population & 16.161 & 2 & 8.081 & 126.2 & $<0.0001 *$ \\
\hline Species & 1.598 & 1 & 1.598 & 15.92 & $0.0001 *$ \\
\hline \multicolumn{6}{|c|}{ Seed Length/Width 2} \\
\hline Population & 142.38 & 2 & 71.19 & 165.92 & $<0.0001 *$ \\
\hline Species & 18.93 & 1 & 18.93 & 25.67 & $<0.0001^{*}$ \\
\hline \multicolumn{6}{|c|}{ Seed Width 1/Width 2} \\
\hline Population & 6.560 & 2 & 3.280 & 48.44 & $<0.0001 *$ \\
\hline Species & 0.826 & 1 & 0.826 & 10.08 & $0.0016^{*}$ \\
\hline \multicolumn{6}{|c|}{ Seed Weight } \\
\hline Population & 0.023150 & 2 & 0.011575 & 44.116 & $<0.0001^{*}$ \\
\hline Species & 0.016446 & 1 & 0.016446 & 59.0474 & $<0.0001 *$ \\
\hline \multicolumn{6}{|c|}{ Seeds in fruit } \\
\hline Population & 2.047 & 2 & 1.023 & 4.241 & $0.0153 *$ \\
\hline Species & 0.005 & 1 & 0.005 & 0.020 & 0.8870 \\
\hline
\end{tabular}


Tab. 3. $p$ values from Tukey post-hoc test for variability of fruits of Berberis croatica in Vošac and B. vulgaris in Rakov Potok and Pharmaceutical botanical garden »Fran Kušan « (PBG) populations. Asterisks indicate significant $p$ values at $p \leq 0.05$.

\begin{tabular}{|c|c|c|c|c|c|}
\hline Population & Vošac & Rakov Potok & Population & Vošac & Rakov Potok \\
\hline & Length & & \multicolumn{3}{|c|}{ Length/Width } \\
\hline Vošac & & & Vošac & & \\
\hline Rakov Potok & $<0.0001 *$ & & Rakov Potok & 0.1867 & \\
\hline PBG & $<0.0001^{*}$ & $0.0001 *$ & PBG & 0.9058 & 0.0757 \\
\hline & Width & & & Weight & \\
\hline Vošac & & & Vošac & & \\
\hline Rakov Potok & $<0.0001^{*}$ & & Rakov Potok & $<0.0001^{*}$ & \\
\hline PBG & $<0.0001 *$ & 0.4388 & PBG & $<0.0001^{*}$ & 0.9738 \\
\hline
\end{tabular}

ANOVA of seed traits showed similar results (Tab. 4). In general, seeds of B. vulgaris were significantly longer, wider and heavier than fruits of B. croatica. Interspecies and

Tab. 4. $p$ values from Tukey post-hoc test for variability of seeds of Berberis croatica in Vošac and B. vulgaris in Rakov Potok and Pharmaceutical botanical garden »Fran Kušan « (PBG) populations. Asterisks indicate significant $p$ values at $p \leq 0.05$.

\begin{tabular}{|c|c|c|c|c|c|}
\hline Population & Vošac & Rakov Potok & Population & Vošac & Rakov Potok \\
\hline \multicolumn{3}{|c|}{ Length } & \multicolumn{3}{|c|}{ Length/Width 2} \\
\hline Vošac & & & Vošac & & \\
\hline Rakov Potok & $<0.0001 *$ & & Rakov Potok & $0.0002 *$ & \\
\hline PBG & $<0.0001 *$ & $<0.0001^{*}$ & PBG & $<0.0001 *$ & $<0.0001^{*}$ \\
\hline \multicolumn{3}{|c|}{ Width 1} & \multicolumn{3}{|c|}{ Width 1 /Width 2} \\
\hline Vošac & & & Vošac & & \\
\hline Rakov Potok & $<0.0001 *$ & & Rakov Potok & $0.0198 *$ & \\
\hline PBG & $<0.0001 *$ & $0.0058^{*}$ & PBG & $0.0004 *$ & $<0.0001^{*}$ \\
\hline & Width 2 & & & Weight & \\
\hline Vošac & & & Vošac & & \\
\hline Rakov Potok & $<0.0001 *$ & & Rakov Potok & $<0.0001 *$ & \\
\hline PBG & $0.0031 *$ & $<0.0001 *$ & PBG & $<0.0001 *$ & $<0.0001^{*}$ \\
\hline & ngth/Width & & & eeds in fru & \\
\hline Vošac & & & Vošac & & \\
\hline Rakov Potok & $0.0008^{*}$ & & Rakov Potok & 0.4001 & \\
\hline PBG & $0.0163 *$ & $<0.0001 *$ & PBG & 0.9030 & 0.6651 \\
\hline
\end{tabular}


intraspecies variability for seed traits was something higher than for fruit traits. The least inter- and intraspecies variability was recorded for the number of seeds in fruit.

\section{Discussion}

The least variable traits in this analysis were fruit and seed length while the most variable traits were fruit and seed weight. That was expected since dimensionality considerations predict that, all else being equal, the coefficients of variability (CVs) of mass-related traits will be larger than those of linear or surface measurements (LANDE 1977), such as length and width of fruits and seeds. The largest variability for fruit and seed weight was also reported by OBESO and HERRERA (1994) which state CVs for these traits ranging generally from 20-30 \% for eight investigated species, including Berberis hispanica. The same authors also reported fruit length and width variability ranging generally between 5 and 12 $\%$ (7.6\% and $7.4 \%$ in B. hispanica for fruit length and fruit width, respectively). These results are in keeping with the results reported in this paper.

Regarding fruit traits of B. vulgaris, AKBULUT et al. (2009) reported much lower values for the wild population of $B$. vulgaris growing in Turkey. For example, average fruit length, width and mass are reported to be $7.69 \mathrm{~mm}, 3.32 \mathrm{~mm}$, and $0.07 \mathrm{~g}$, (compared with the average value range for the same traits in this investigation, that is, $10.20-11.29 \mathrm{~mm}, 5.29-5.83$ $\mathrm{mm}$ and $0.1602-0.2199 \mathrm{~g}$ ). This could be explained by environmental factors but also by great intraspecific variation in Berberis already reported by LANDRUM (1999).

Generally, fruits and seeds of B. vulgaris were significantly longer, wider and heavier than fruits and seeds of B. croatica. This is in congruence with the results of KARLOVIĆ et al. (2009) who, when analyzing distinguishing morphological traits between these two species, stated that $B$. croatica is characterized by reduced growth/size traits compared with $B$. vulgaris. Our results support this thesis. Results regarding fruit and seed traits show significant differences between species for most analyzed traits (10 out of 12). Nevertheless, whether the observed differences between $B$. croatica and B. vulgaris are merely non-hereditary modifications caused by environmental factors or species-related differences, should be further tested by means of genetic analysis.

\section{Acknowledgements}

This work was supported by the Ministry of Science, Education and Sports of the Republic of Croatia (project »Micromorphological and chemotaxonomic researches on some species of the family Lamiaceae $\ll)$.

\section{References}

Akbulut, M., Calisir, S., Marakoglu, T., Coklar, H., 2009: Some physicomechanical and nutritional properties of barberry (Berberis vulgaris L.) fruits. Journal of Food Process Engineering 32, 497-511.

Allen, R. B., Wilson, J. B., 1992: Fruit and seed production in Berberis darwinii Hook., a shrub recently naturalised in New Zealand. New Zealand Journal of Botany 30, 45-55. 
HENDRIX, S. D., Sun, I. F., 1989: Inter- and intraspecific variation in seed mass in seven species of umbellifer. New Phytologist 112, 445-451.

HERRERA, C. M., 1992: Interspecific variation in fruit shape: allometry, phylogeny and adaptation to dispersal agents. Ecology 73, 832-1841.

Herrera, C. M., 1998: Population-level estimates of interannula variability in seed production: what do they actually tell us? Oikos 82, 612-616.

ERIKSSON, O., 1999: Seed size variation and its effect on germination and seedling performance in the clonal herb Convallaria majalis. Acta Oecologica 20, 61-66.

JORDANO, P., 1984: Seed weight variation and differential avian dispersal in blackberries Rubus ulmifolius. Oikos 43, 149-153.

KARlović, K., KREMER, D., LiBER, Z., ŠATOVIĆ, Z., VRŠEK, I., 2009: Intra- and interpopulation variability and taxonomic status of Berberis croatica Horvat. Plant Biosystems 143, 40-46.

LANDE, R., 1977: On comparing coefficients of variation. Systematic Zoology 26, 214-217.

LANDRUM, L. R., 1999: Revision of Berberis (Berberidaceae) in Chile and Adjacent southern Argentina. Annals of the Missouri Botanical Garden 86, 793-834.

Obeso, J. R., Herrera, C. M., 1994: Inter and intraspecific variation in fruit traits in co-occuring vertebrate dispersed plants. International Journal of Plant Science 155, 382-387.

Rudolf, P. O., 1974: Berberis, barberry, mahonia. In: SchopmeYer, C. S. (tech. coord.), Seeds of woody plants of the United States, 247-251. USDA Forest Service, Washington, DC.

Zovko Končić, M., Kremer, D., Karlović, K., Kosalec, I., 2010: Evaluation of antioxidant activities and phenolic content of Berberis vulgaris L. and B. croatica Horvat. Food and Chemical Toxicology 48, 2176-2180.

Willson, M. F., Michaels, H. J., Bertin, R. I, Benner, B., Rice, S., Lee, T. D., HARTGERINK, A. P., 1990: Intraspecific variation in seed packaging. American Midland Naturalist 123, 179-185. 